\title{
Point Cloud accuracy of Smartphone Images: Applications in Cultural Heritage Environment
}

\author{
Costantino D. ${ }^{1}$, Pepe M. ${ }^{1}$, Alfio V.S. ${ }^{1}$ \\ ${ }^{1}$ DICATECh, Politecnico di Bari, via Orabona 4, 70126 Bari, Italy \\ (domenica.costantino, massimiliano.pepe, vincenzosaverio.alfio)@ poliba.it
}

\begin{abstract}
The 3D reconstruction of objects or structures belong to Cultural Heritage is a central theme in the field of conservation and restoration. In this context, photogrammetry with low-cost sensors represents an opportunity to survey in an easy, simple, accurate and economic way the immense Cultural Heritage. The aim of this research is to evaluate the achievable level of accuracy of the point cloud obtained from images generated by smartphones and processed in the Structure From Motion (SfM) and Multi View Stereo (MVS) environment. To validate the method of analysis, three case studies of structures belonging to the Cultural Heritage and present in southern Italy are shown in the paper. In particular, a comparison between the point clouds obtained by the smartphones and the point cloud obtained by digital reflex cameras and Terrestrial Laser Scanner was carried out. The results show a difference between the point clouds in the order of a few millimeters and, of consequence, the enormous potential of the smartphones in photogrammetry field.
\end{abstract}

Key words: Smartphone, photogrammetry, terrestrial survey, SfM, MVS.

\section{INTRODUCTION}

The use of digital images obtained from low-cost sensors for photogrammetric purposes has become increasingly massive due to recent develop of the software based on Structure from Motion (SfM) and Multi View Stereo (MVS) algorithms that allows to obtain three-dimensional models. In particular, SfM approach requires, for camera models based on collinearity equations, a minimum of three corresponding points per image acquired according to convergent angles [1]. As result, it is necessary to capture with high image overlap to cover an object or structure. The MVS approach allows increasing the density of points starting from the point cloud generated in SfM process. In this way, it is possible to build a dense point cloud of an object or structure. A revolutionary aspect of using this approach has been the use of low-cost cameras. Low-cost cameras are characterized by less geometric stability than professional cameras; of consequence, this problem leads to lower reliability and durability of the internal camera geometry modelling [2]. To overcome this situation, algorithms and quick calibration procedures have been developed in recent years that allow these cameras to be used for 3D measurement purposes [3, 4]. Therefore, after having estimated estimate the intrinsic parameters of the camera, such as the focal length (f) of the lens, the coordinates of the centre of projection of the image (xp, yp) and the radial lens distortion coefficients $(\mathrm{k} 1, \mathrm{k} 2, \mathrm{k} 3)$ it is possible to produce 3D model $[5,6]$.

Various sensors are available on the market, such as Compact System Camera (CSC), inexpensive dSLR (digital Single-Lens-Reflex) cameras, bridge camera, action cameras, smartphone-equipped cameras, etc. There are several criteria for choosing a low cost camera for photogrammetry: high resolution, good quality lens, prime/fixed lens (a lens without zoom and that has one fixed focal length), some control over settings. The smartphones can be a good choose since that they are able to acquire images in high resolution and most have a prime/fixed lens.

The smartphone photogrammetry was applied in many filed of application: Micheletti et al., 2013 [7] investigate the geomorphological potential of freely available and accessible structure $\square$ from $\square$ motion photogrammetry using a smartphone. In this latter case study, the images acquired with the camera included Apple iPhone 4 and processed in SfM approach allowed to obtain a centimetre precision on the determination of DTM on Alpine area. Barbero-García et al., 2017 [8] use low-cost smartphone-based photogrammetry for the analysis of cranial deformation in infants. The results show that the correspondence between the manual and the photogrammetric 3D model measurements was high as far as head marks are available, with differences of $2 \mathrm{~mm} \pm 0.9 \mathrm{~mm}$; without marks, measurement results differed up to $20 \mathrm{~mm}$. Costanzo et al., 2017 [9] proposed a system based on the use smartphone that is useful during the emergency management and for preserving memories of local communities. Mnati et al., 2019 [10] discussed about the use of the smartphone and apps in the human movements; indeed, the aim of this latter research is to evaluate the accelerometer data by comparing the captured data with camera image-based photogrammetric data. 
Research and studies on cultural heritage are limited; this is due to the high and conservative standards required for the survey and representation of the structures. In this context, Kirchhöfer et al., 2001 [11] wrote about the integration of camera in smartphone and other sensors with the aim to test the performance of the recording system at a real heritage site St. Catherine's Oratory, in United Kingdom. Shults (2017) [12] discussed about the use of the smartphone in Cultural Heritage environment. In order to build the 3D model, the images were acquired by the use of Meizu M3 Max, which is a smartphone designed and produced by the Chinese manufacturer Meizu. The results of this latter experimentation shown encouraging accuracy values and, of consequence, the model obtained by IBM method meets the requirements of archaeological and historical measurements. Boboc et al., 2018 [13] have examined the reliability of smartphones that incorporate 3D depth sensors for 3D reconstruction of cultural heritage objects. Yilmazturk and Gurbak (2019) [14] discussed about some experimentation carried out on 3D test field using more smartphone. In addition, the authors wrote about a survey of a historical structure performed by the use of Galaxy S4 smartphone camera; the post-processing of the images generated by smartphone camera in SfM-MVS environment shown how the smartphone cameras can be easily used in photogrammetric applications. Russo et al., 2019 [15] discussed about the survey of the church of San Rocco, framed in Campo Marzio district in Rome (Italy) carried out by the use of OnePlus 6 smartphone; this device allows to capture images with a resolution of 16 megapixels.

\subsection{Motivation}

In recent years, digital camera sales have been falling [16]. In this context, Smartphone camera technology has come on leaps and bounds in recent years. According to InfoTrends' 660 trillion photos were taken worldwide in 2013, 810 billion in 2014, 1 trillion in 2015, 1.1 trillion in 2016 and 1.2 trillion in 2017. The $85 \%$ of all pictures taken in 2017 will be captured on smartphones. This huge amount of photos is to be found in several factors, among which the most important is the ability to have a device always close to the user. Indeed, the award-winning photographer Chase Jarvis once coined the phrase "the best camera is the one that's with you". In addition, another reason for the great development of smartphones is due to the increasingly competitive costs of these devices compared to other image capture technologies. In November 2000, Sharp Corporation launched the first camera phone, J-SH04 with a standard resolution of 0.3 Mega-pixel (MP). It was the first Japanese mobile phone to be produced with an integrated camera with CMOS sensor and a color display.

In 2018, the Huawei P20 smartphone amazed everyone with its incredible 40-megapixel photo sensor. During the year, even a smartphone with a 108 megapixel main lens was developed (Xiaomi Mi Note 10). It is easy to predict that 108 megapixels will become the norm in 2020 , so much so that Samsung has already announced a 144megapixel sensor while Qualcomm's latest SoC, the Snapdragon 865, which will be the processor of choice for top-of-the-range mobile phones next year, is capable of supporting photographic lenses of up to 200 megapixels. The graph in Figure 1 shows the development over the years of two dSLRs (Canon's EOs and Nikon's D series) and some smartphone series (Huawei P Series, iPhone models, Xiaomi models and Samsung $\mathrm{S}$ Series). As can be seen from the graph (Figure 1), while dSLR cameras show a constant trend with a slight slope, smartphones show an exponential trend. Among the smartphone examined, the only one that does not show this trend is the iPhone. In addition, Smartphone manufacturers present new models on an annual basis and, as a result, very often result in an improvement in the quality of the sensor optics.

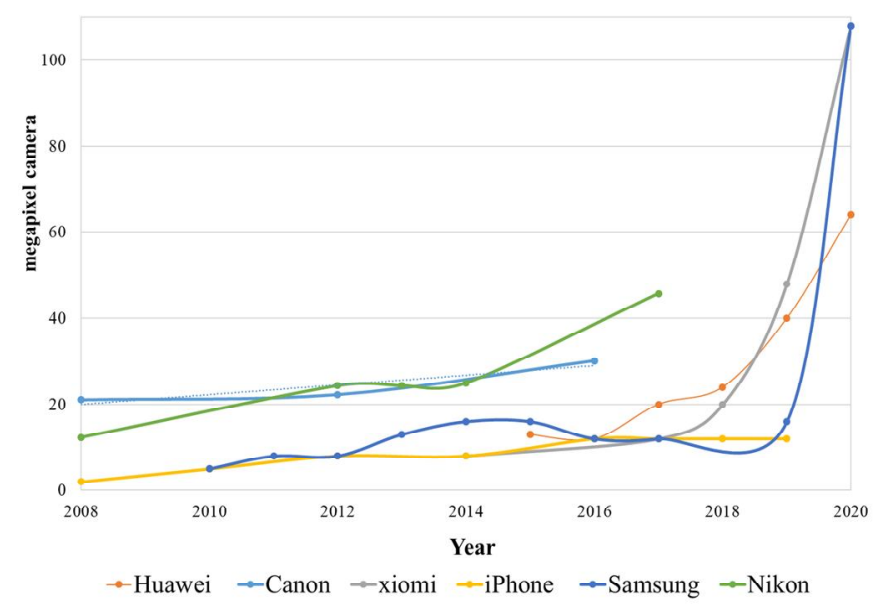

Figure 1: Evolution of sensors over the years

\subsection{Smartphone built-in camera capabilities}

When an image is being captured by a camera, light passes through the lens and falls on the image sensor. The image sensor consists of picture elements, also called pixels that register the amount of light that falls on them. They convert the received amount of light into a corresponding number of electrons. The stronger the light, the more electrons are generated. The electrons are converted into voltage and then transformed into numbers by means of an $\mathrm{A} / \mathrm{D}$ converter. The signal constituted by the numbers is processed by electronic circuits inside the camera. Actually, there are two main technologies that can be used for the image sensor in a camera, i.e. CCD (Charge-coupled Device) and CMOS (Complementary Metal-Oxide Semiconductor).

The CCDs are sensors based on an array of passive photodiodes that integrates charge during the exposure time of the camera. The charge is then transferred to common electronics which reads the accumulated charges of the different pixels and translates them in voltages. Since the 
CCD is a passive pixel device (i.e. with no electronics at pixel level) the quantum efficiency is very high: this is an advantage in applications where the light is quite poor.

The CMOS are sensors based on an array of active pixel: the pixel-level electronics (typically 3 or 4 transistors) translates the charge accumulated in the photodiode in a well-defined voltage; in this way, the output of each pixel needs only to be acquired and sampled [17, 18].

Although CMOS sensors were developed after CCD sensors, they soon gained a significant market share. Thanks to recent technological developments, the maturity of CMOS detectors is now well established and the advantages of their specific features, allowed by in-pixel amplification, column-parallel architecture and the use of deep sub-micron CMOS processes, are currently used in several applications [19]. One of their strengths is undoubtedly their small size which has allowed their introduction in various devices, such as smartphones. The technological development of these sensors is constantly and rapidly evolving.

\section{METHOD AND DATA}

Using the images produced by camera device in SfM/MVS software, a dense and colored point clouds can be obtained $[20,21]$. In order to verify the geometric accuracy of the point cloud, it is possible to make a comparison in two ways. In fact, it is possible to make a first comparison with the point cloud generated in SFM/MVS environment and dSLR cameras equipped with stabilized optics of good quality. For the several case studies, Nikon D3300 dSLR camera with a focal length of $18 \mathrm{~mm}$ was used. This latter camera has a 24.2 megapixel CMOS sensor; in addition, the camera's ISO range tops out at 25,600 and continuous shooting up to $5 \mathrm{fps}$.

Besides a comparison between point clouds generated by passive sensors, it is possible to make a further comparison with the point cloud generated with a Terrestrial Laser Scanner (TLS). The pulsed scanner used in the experiment described in the several case studies is the Leica HDS3000. The company claims a precision of $6 \mathrm{~mm}$ at a scan distance of $50 \mathrm{~m}$. The maximal scan distance amounts to $100 \mathrm{~m}$. The scan range reaches $270^{\circ}$ in the vertical direction and is complete, $360^{\circ}$, in the horizontal plane. Additionally, to the xyz-location of the reflecting surface point, the intensity of the received echo is registered as well [22].

The pipeline summarizing the method developed to assess the accuracy of the point cloud obtained using images generated by smartphones and software based on the use of SfM-MVS software is shown below (Figure 2).

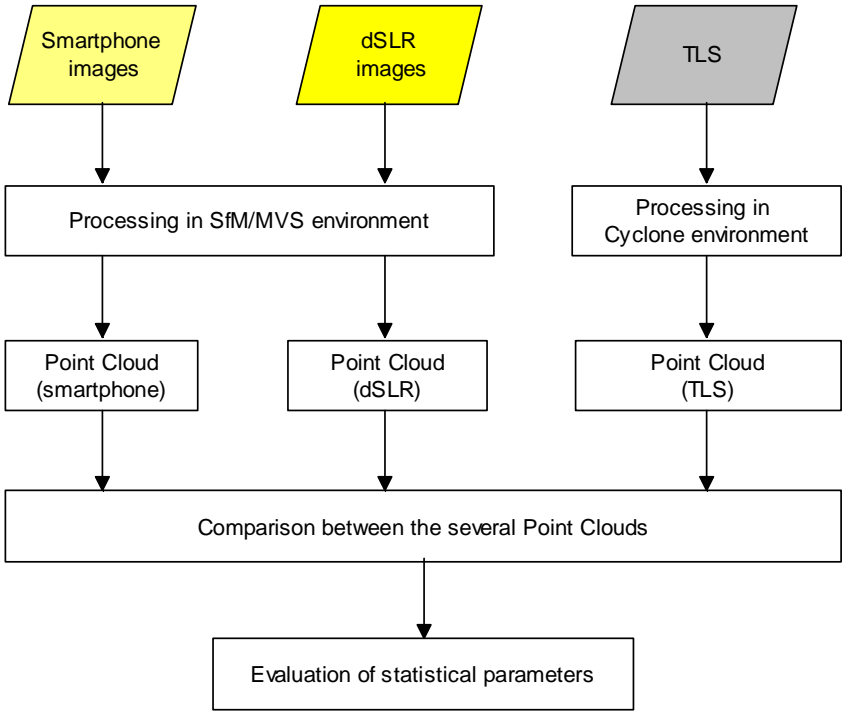

Figure 2: Pipeline of the method developed

To validate the method, three case studies in Cultural Heritage environment are shown in the paper. The first case study concerns the potentiality in the use of the images generated by the Xiaomi Redmi Note 7 smartphone for the survey in rather narrow and low light environments; the survey was carried out inside an ancient rock church of very recent discovery. In the second case, the roof of a church located in Martina Franca, South of Italy was modelled using iPhone X smartphone. In the third case, the survey of the dome of a church located was carried out using Huawei P20 lite. The three study areas are located in southern Italy (Figure $3 a)$ : the first and the third are located in the municipality of Grottaglie and the second in the municipality of Martina Franca, as shown in figure $3 b$.

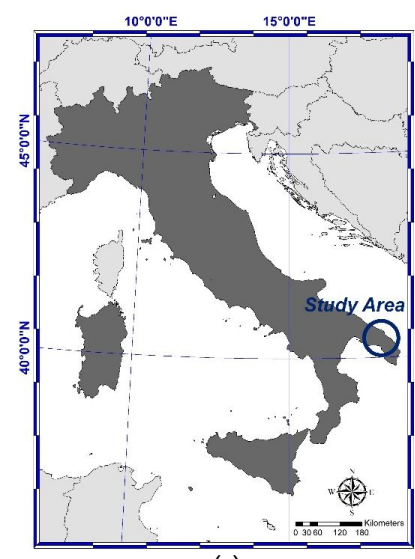

(a)

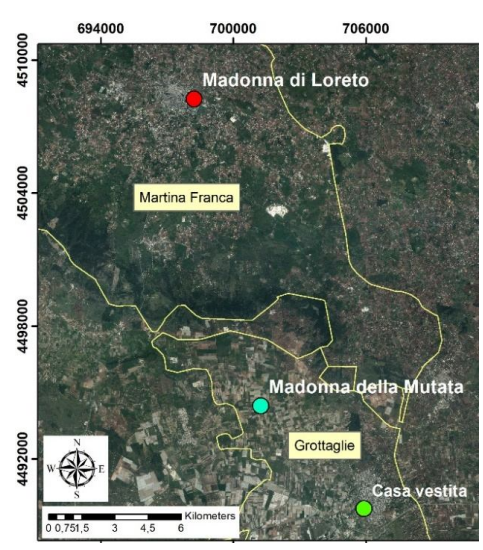

(b)
Figure 3: Identification of the study area on national scale (a) and local scale (b)

However, before starting the geomatics survey on the three case studies, a preliminary analysis of the photogrammetric accuracy of the sensors tested on a 3D test-field is necessary. In this way, it is possible to identify the level of accuracy achievable by commercial smartphones in the survey of Cultural Heritage applications. 


\subsection{Assessment of the photogrammetric quality of some tested smartphones on 3D test-field}

In order to identify the level of photogrammetric accuracy achievable with different smartphones and cameras, a 3D test-field was realized. In particular, 18 Ground Control Points (GCPs) were positioned in different positions.

The GCPs was surveyed by a Reflectorless Electronic Distance Measurement (REDM) Leica Viva TS11 total Station.

The images acquired by several cameras was processed by the use of 3DF Zephyr SfM-MVS software, which is developed and marketed by the Italian software house 3DFLOW.

The acquisition scheme, realized according to the principles of the SfM and MVS approach, is shown below (Figure 4).

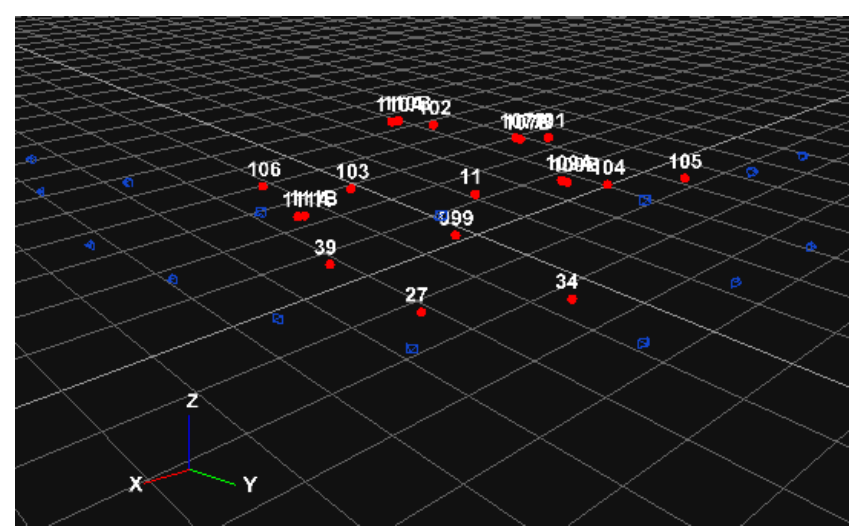

Figure 4: Camera positions (shown in blue) and spatial configuration of the GCPs (red dots)

The datasets of the images acquired by Nikon D3300 with fisheye lens was processed in Agisoft Metashape, which is developed by Agisoft LLC located in St. Petersburg in Russia. The error was evaluated on 8 Check Points (CPs). The results (Mean and Standard Deviation - StD) obtained using the several datasets of images are summarized in the Table 1, where it easily sees that the accuracy achieved by smartphones is close to that of dSLR cameras.

Table 1: Accuracy evaluation on 3D test field

\begin{tabular}{|l|c|c|}
\hline \multirow{2}{*}{} & \multicolumn{2}{|c|}{ Error } \\
\cline { 2 - 3 } & Mean (m) & StD (m) \\
\hline Huawi P20 lite & 0.004 & 0.005 \\
\hline iPhone X & 0.007 & 0.005 \\
\hline Xiaomi Redmi note 7 & 0.004 & 0.004 \\
\hline Nikon D3300 (f=18mm) & 0.002 & 0.001 \\
\hline Nikon D3300 (fisheye f=10mm) & 0.001 & 0.001 \\
\hline
\end{tabular}

\subsection{Case study 1: Survey and modelling of a rock church using Xiaomi Redmi Note 7}

\subsubsection{Historical notes}

The rock church is located in the ceramic district of Grottaglie in the province of Taranto and discovered in 2008 during the refurbishment of the garden of Casa Vestita. The cult building was hidden behind an oven used for cooking food, never used and partially collapsed, hidden by a filling of earth and stones.

The church, which can be dated between the end of the 13th century and the beginning of the 14th century on the basis of the materials found inside, has a very simple plan with a single nave ending in three rectangular apsides on the eastern side. In order to access it from the garden, it is necessary to descend a few large ones as it is subject to a floor level of about 2.00 meters.

The apsides are placed inside an architectural backdrop consisting of three arches resting on four pseudo twisted columns. On the ceiling in correspondence of the altar appears a circular engraving with a cross in relief inside; this element, which has the function of differentiating the liturgical space, finds numerous comparisons in other contexts of Taranto and Lecce (see Figure 5).

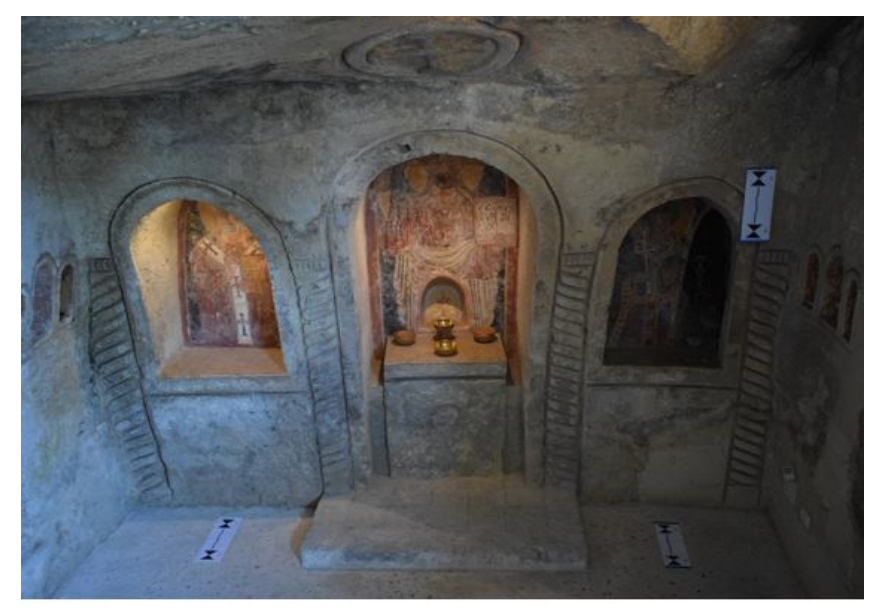

Figure 5: Interior of the rock church

The side apsides represent St. Nicholas and St. Barbara, while the central apses represent a blessing Christ with an open book in his left hand. The style of the frescoes recalls the pictorial taste that characterized the Apulian production in the 13th century in the territory of the then Principality of Taranto.

\subsubsection{Instrumentation and survey phase}

The survey of the church was carried out with two different types of camera: reflex camera Nikon D3300 and smartphone Xiaomi Redmi Note 7 with 9 MP camera setting. In order to obtain a perfectly scaled three-dimensional model, 8 numbered targets were placed inside the church that contained a $0.20 \mathrm{~m}$ metric bar useful for a final evaluation of the perfect scaling of the model. The arrangement of the targets took into account the irregular shape of the church so they were placed not only on the floor but also on the irregular surfaces that characterize the church.

The photogrammetric surveys were carried out in parallel axes on the surfaces with an almost regular course such as the apse that contains the frescoes and the side walls, 
guaranteeing in any case an adequate degree of overlap between the frames. Moreover, in order to represent the frescoes and other valuable elements contained inside the crypt in more detail, many other photos were acquired that were useful in the post-processing process.

In order to taking into account of the low light conditions of the surrounding environment during the photogrammetric survey, the camera was supported by a tripod and the focal length of $18 \mathrm{~mm}$ and an ISO sensitivity of 1600 with a fixed exposure time of 1/60 s was kept constant and 186 images were recorded.

As for the photogrammetric survey performed with the smartphone, it was necessary to take into account the shorter focal length than that of the dSLR (focal length of about 5 $\mathrm{mm})$. In this way, 238 frames were captured.

The photogrammetric 3D modelling software used for the several tests is 3DF Zephyr. The images were processed by setting "Highest" in the definition of keypoint density and an "Accurate" mode was chosen in the matching type. Highest quality settings were also used in the reconstruction of the dense cloud.

For the scaling of the model, the 16 GCPs were identified, suitably positioned inside the church, and for each pair a "Sample Size" of $0.30 \mathrm{~cm}$ was identified.

Details of reconstruction of the dense point cloud obtained by two different photographic datasets are shown in the Figure 6; in particular, Figure 6 (a) shows the result obtained with the dSLR camera while Figure 6 (b) shows the result obtained by the smartphone. In both cases, it is possible to observe a high definition of the point cloud when describing the complex geometry of the object.

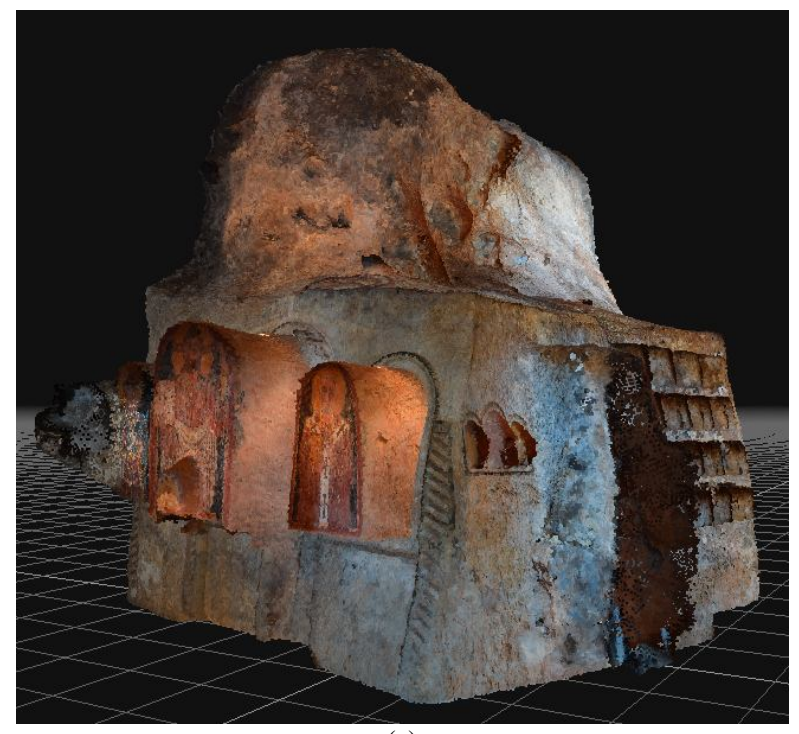

(a)

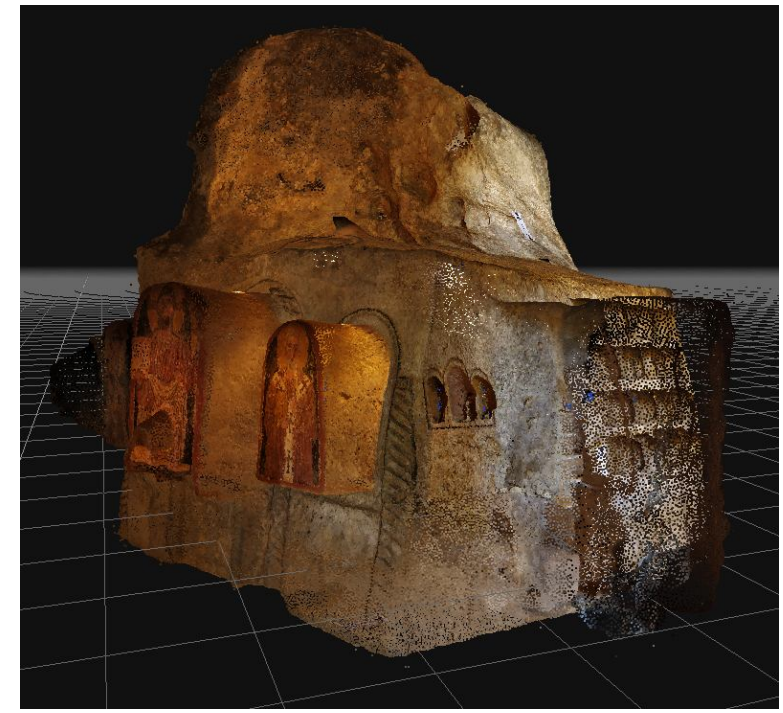

(b)

Figure 6: Dense point cloud: dataset obtained from dSLR Nikon D3300 (a); dataset acquired by Xiaomi Redmi Note 7 smartphone

(b)

\subsubsection{Comparison of results}

A first element taking into consideration concerns the number of points obtained in the reconstruction of the dense cloud datasets. The values shown in Table 2 describe the results obtained during the processing of the two different photographic datasets within the 3DF Zephyr software.

Table 2: Result of post-processing in 3DF Zephyr

\begin{tabular}{|r|c|c|}
\hline & dSLR & $\begin{array}{c}\text { Xiaomi Redmi } \\
\text { Note 7 }\end{array}$ \\
\hline $\begin{array}{r}\text { Numbers of image } \\
\text { [\#] }\end{array}$ & 186 & 239 \\
\hline Tie Points [\#] & 165.375 & 163.826 \\
\hline Dense cloud [\#] & 7.821 .677 & 3.603 .357 \\
\hline
\end{tabular}

After the elaboration of the two dense clouds, they were overlapped to determine which deviation could be generated in the elaboration of the two different photographic datasets. The merge process of the two different clouds, was processed by the same 3DF software Zephyr: through a simple drag and drop operation, the dataset obtained from the smartphone sensor was imported and, subsequently, the fusion of the two clouds was performed.

The registration of the two different datasets was carried out using suitable GCPs. The comparison of the point cloud shows a residual max of $0.001 \mathrm{~m}$ and average distance of 0.4 $\mathrm{mm}$. Starting from an algorithm of comparison of the Euclidean distance point to point, it has also been reconstructed the histogram where on the y-axis was represented the number of points analyzed and on the abscissae axis the maximum residue obtained during the comparison between the clouds of dense points. From the evaluation of the histogram (Figure 7), it was noted that most of the points analyzed fall within the error range of about 5 $\mathrm{mm}$, while coarser errors are gradually lower and in any case 
not too much higher than one centimeter when considering a maximum sampling interval.

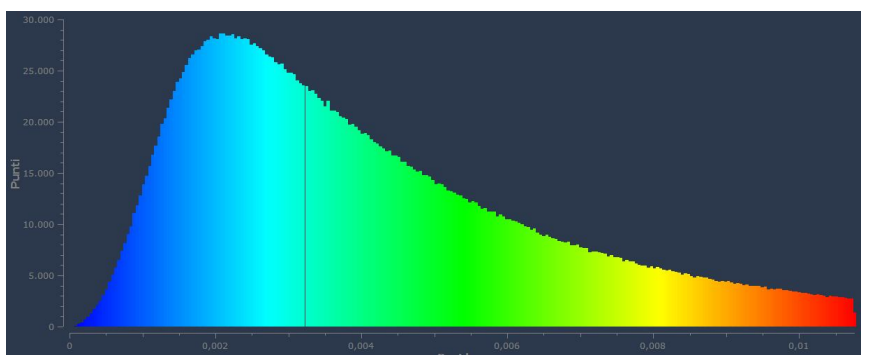

Figure 7: Histogram of difference: difference of the point clouds obtained by the images generated by dSLR camera and Xiaomi

Redmi Note 7 smartphone (Mean: 0.003m; StD:0.002m)

\subsubsection{TLS and photogrammetric survey: geometric comparison}

Scans were carried out from three stand-points, placed respectively at a distance ranging from 1 to $5 \mathrm{~m}$ from the walls of the church.

The post processing of TLS data was carried out by Cyclone software.

The merging of the three scans was performed using 6 GCPs; the error on the individual GCPs was less than $1 \mathrm{~cm}$. In this way, after the elimination of some points considered outliers, a point cloud of 1,758,729 points was generated, as shown below (Figure 8).

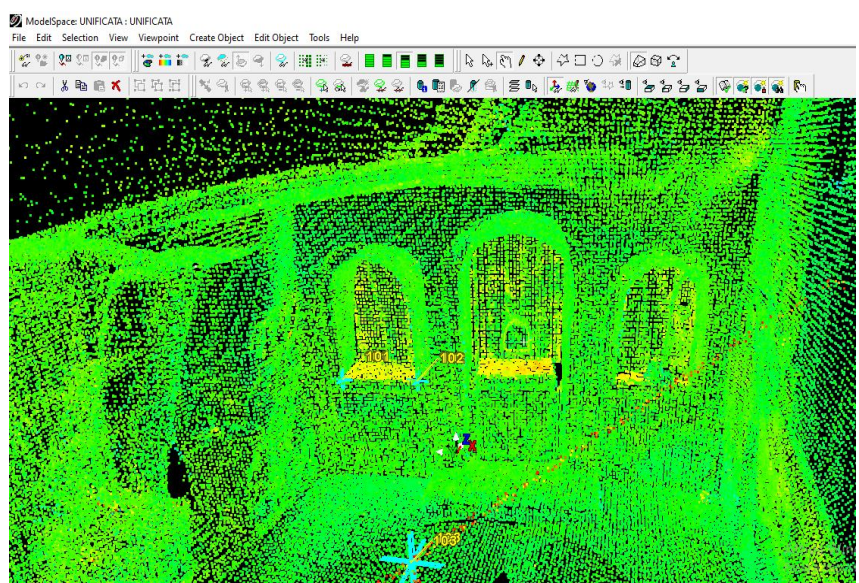

Figure 8: Viewing of the point cloud generated by TLS in Cyclone.

This point cloud generated by TLS was compared to that obtained with photogrammetric data (Figure 9), i.e. with the point cloud obtained by smartphone (Figure 9a) and dSLR images (Figure 9b).

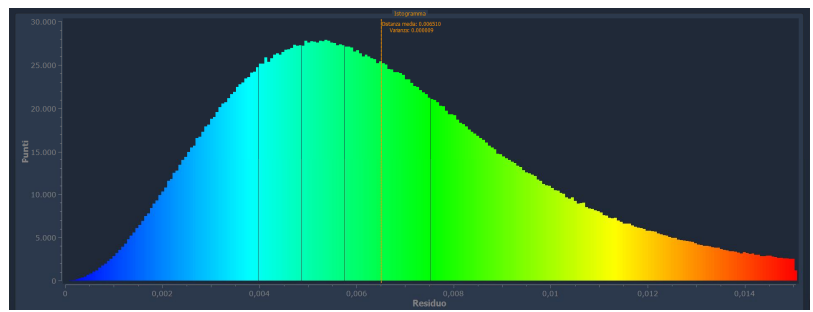

(a)

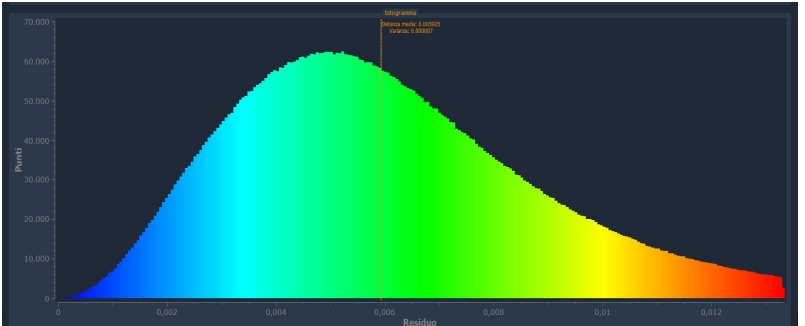

(b)

Figure 9: Point cloud comparison: TLS - Images generated by Xiaomi Redmi Note 7 (Mean: 0.006 m; StD:0.001 m)

(a); TLS- Images generated by Nikon D3300 ( $\mathrm{f}=18 \mathrm{~mm})$ (Mean: $0.006 \mathrm{~m}$; StD: $0.001 \mathrm{~m})(\mathrm{b})$

\subsection{Case study 2: Survey and modelling of the church of Madonna di Loreto using iPhone $\mathbf{X}$}

\subsubsection{Brief history of the site}

The church of the Madonna di Loreto, located in Martina Franca town, is adjacent to that of San Michele and has a very similar history. In fact, both date back to the fourteenth century and both underwent major alterations over the centuries, until 1984, when they were recovered by priests Stefano Castellana and Martino Bello. The ceiling is decorated with tempera; the predominant colors are blue and brown.

\subsubsection{Photogrammetric and TLS surveys}

The survey of the church was carried out using iPhone $\mathrm{X}$ smartphone. To cover the entire ceiling and some side parts of the church 761 photos were taken. The datasets were processed by the use 3DF Zephyr; the point cloud of the church ceiling is shown below (Figure 10)

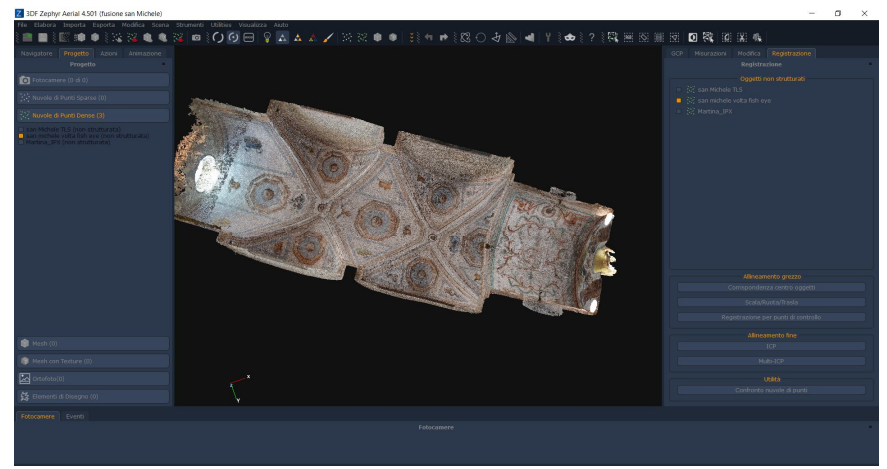

Figure 10: Viewing of the dense point cloud in 3DF Zephyr software

The same survey of the church ceiling was performed by the use of Nikon D5000 dSLR camera and fish-eye lens; in this way, 97 photos was carried out and elaborated with 3DF Zephyr software. In addition to the photogrammetric survey, a TLS survey was performed; in particular, using HDS3000 TLS, five scans were carried out. The merging of scanning and point cloud editing led to the formation of about $2,165,854$ million points. The comparison between the point clouds generated in several ways is shown below. 


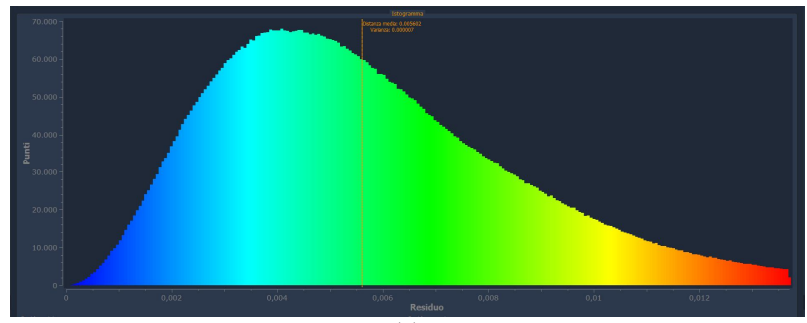

(a)

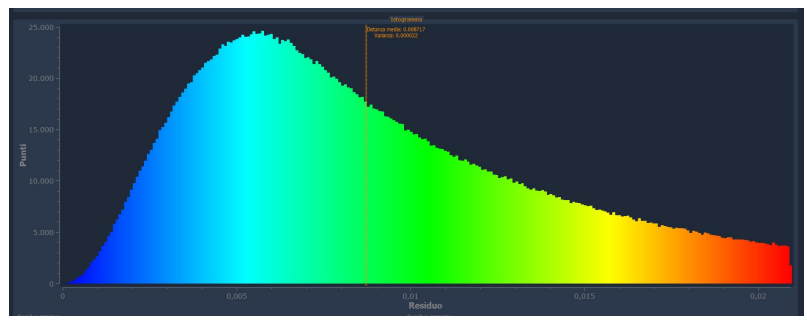

(b)

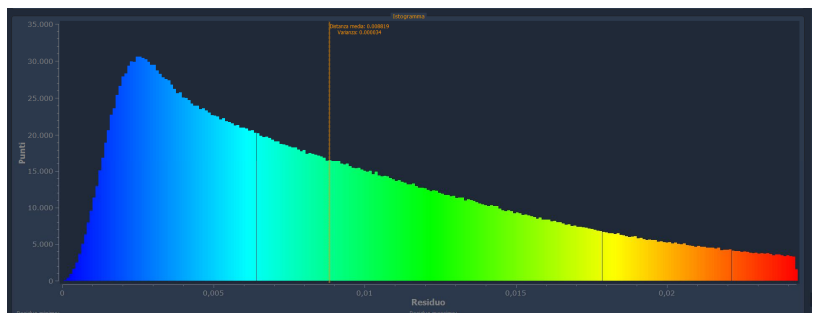

(c)

Figure 11: Comparison of the point clouds: TLS - Images generated by Nikon D5000 with fish-eye lens (Mean: $0.005 \mathrm{~m}$; StD: $0.001 \mathrm{~m}$ ) (a); TLS - Images generated by iPhone X (Mean: $0.008 \mathrm{~m}$; StD: 0.001 m) (b); Images generated by Nikon with fish-eye lens - Images generated by iPhone X (Mean: $0.009 \mathrm{~m}$; StD: $0.001 \mathrm{~m}$ ) (c).

\subsection{Case study 3: Survey and modelling of the dome of Sanctuary of Madonna di Mutata using Huawei P20 lite}

\subsubsection{Historical notes}

A few kilometers from the town of Grottaglie (Italy) the early Christian sanctuary dedicated to the Assumption of the Virgin rose in the heart of what was once the forest of the Taranto area. The church was probably built in the 10th century. In the right aisle, the first span houses the altar with the limestone statue of St. Joseph (1679); a niche houses a Romanesque statue of the Virgin; followed by the altar dedicated to the Mutata with the ancient fresco of the Madonna. From this span rises the dome with fans and paintings. The church is embellished by a beautiful seventeenth-century majolica grottagliese flooring, with geometric decorative elements or leaves and flowers, yellow, sky blue and pink predominate on the white background.

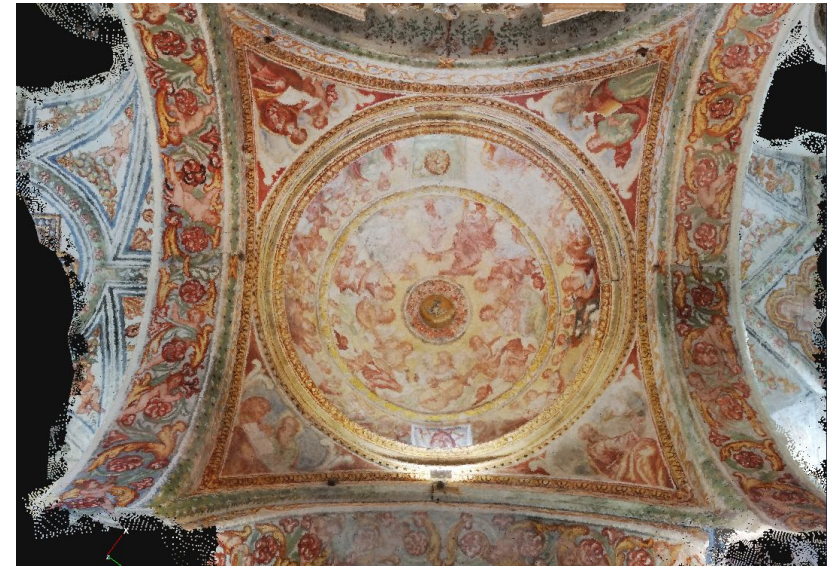

(a)

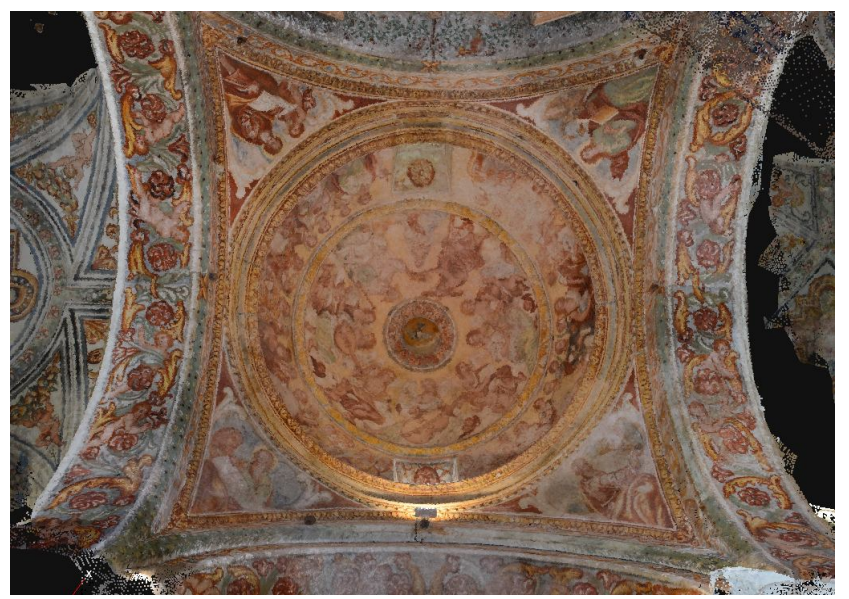

(b)

Figure 12: Viewing of the dense point cloud using images generated by smartphone (a) and by the use of Nikon dSLR.

For the three-dimensional reconstruction of the dome, as in previous case studies, a photogrammetric survey was carried out. In this case study, the Huawei P20lite smartphone and Nikon D3300 with $18 \mathrm{~mm}$ lens were used. In particular, 126 images were acquired by the smartphone and 169 images were acquired by dSLR camera. The 3D reconstruction of the model was realized in SfM/MVS, which led to the construction of a dense cloud point cloud of 1 million points in the case of Smartphone images (Figure 12).

In addition, by positioning the TLS in a central manner with respect the center of the dome, a scan with a spatial resolution of $5 \mathrm{~mm}$ was performed. This scan allowed obtaining a detailed dense point cloud $(1,503,863$ points) of the dome.

The comparison of the several point clouds was carried out in 3DF Zephyr environment. The histograms of the difference between the point clouds obtained by several geomatics surveys are showed below (Figure 13). 


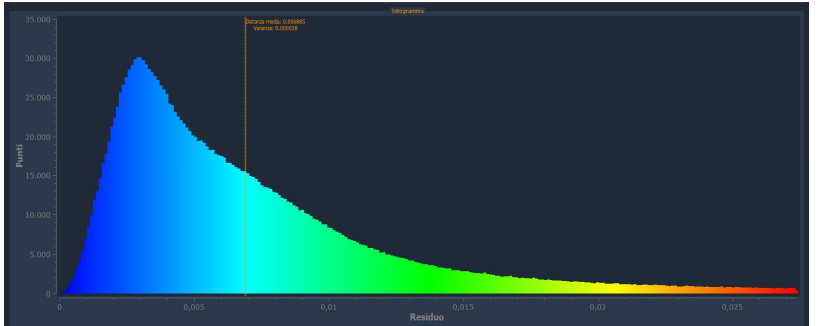

(a)

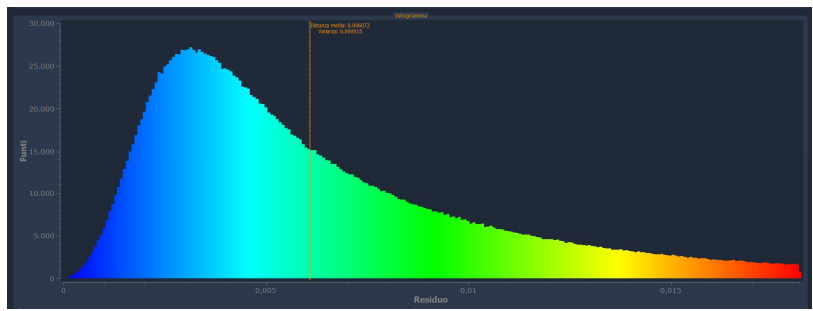

(b)

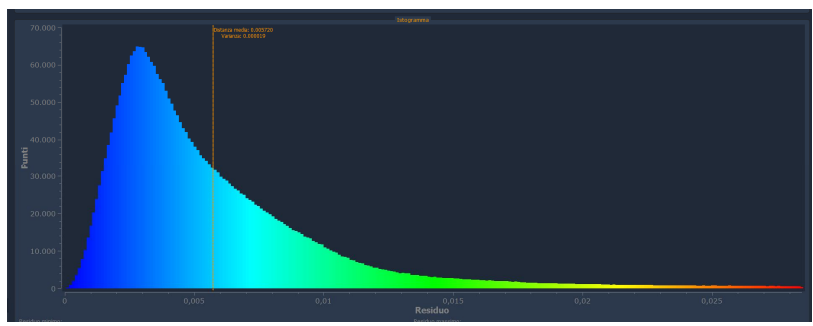

(c)

Figure 13: Comparison of the point clouds: TLS - Images generated by Huawei P20 lite (Mean: 0.006m; StD: 0.001m) (a); Images generated by Nikon with $18 \mathrm{~mm}$ lens - Images generated by Huawei P20 lite (b) (Mean: 0.006m; StD: 0.001m); TLS - Images generated by Nikon D3300 with $18 \mathrm{~mm}$ lens (Mean: $0.005 \mathrm{~m}$; StD: $0.001 \mathrm{~m}$ ) (c)

\section{CONCLUSIONS}

The use of images generated by smartphones and software based on SfM and MVS algorithms have made it possible to obtain a three-dimensional and accurate reconstruction of objects or structures. Three commercial smartphones were taken into account; the point cloud produced by the images generated by smartphones was compared with the photogrammetric one obtained by high-performance sensors, such as dSLR cameras, and with the point cloud generated by a Terrestrial Laser Scanner.

The comparison between the point clouds have showed small difference both in the 3D test-field and three case studies. Especially, the camera supplied in the Huawei P20 lite smartphone has made it possible to obtain high quality point clouds both from a geometric and radiometric point of view. For this reason, the smartphone can be used even in Cultural Heritage environment with the condition of performing the survey with high image overlap and using a smartphone with good optics.

\section{ACKNOWLEDGEMENT}

We want to thank the custodians of the churches for their helpfulness and support during the phases of the survey. This research was carried out in the project: PON "Ricerca e Innovazione" 2014-2020 A. I.2 "Mobilità dei Ricercatori" D.M. n. 407-27/02/2018 (AIM1895471 - Line 1).

\section{REFERENCES}

1. Pepe, M., Fregonese, L. and Crocetto, N. Use of SfM-MVS approach to nadir and oblique images generated through aerial cameras to build 2.5 D map and 3D models in urban areas, Geocarto International, 2019, pp. 1-22.

https://doi.org/10.1080/10106049.2019.1700558

2. Sanz-Ablanedo, E., Rodríguez-Pérez, J. R., Arias-Sánchez, P. and Armesto, J. Metric potential of a 3D measurement system based on digital compact cameras, Sensors, 9(6), 2009, pp. 4178-4194.

3. Karras, G. E. and Dionyssia M. Simple calibration techniques for non-metric cameras, in International archives of photogrammetry remote sensing and spatial information sciences, 34.5/C7, 2002, pp. 39-46.

4. Fraser, C., Automatic Camera Calibration in Close-Range Photogrammetry, Proceedings of the ASPRS 2012 Annual Conference, Sacramento, CA, USA, 19-23 March 2012; pp. 19 - 27.

5. Pepe M., Costantino D., Techniques, Tools, Platforms and Algorithms in Close Range Photogrammetry in Building 3D Model and 2D Representation of Objects and Complex Architectures. Computer-Aided Design \& Applications, 18(1), 2021, pp. 42-65

6. Pepe, M. and Parente, C. Cultural Heritage documentation in SIS environment: an application for "Porta Sirena" in the archaeological site of Paestum, Int. Arch. Photogramm. Remote Sens. Spatial Inf. Sci., Vol. XLII-5/W1, 2017, pp. 427-432.

7. Micheletti, N., Chandler, J. H., and Lane, S. N. Investigating the geomorphological potential of freely available and accessible structure $\square$ from $\square$ motion photogrammetry using a smartphone. Earth Surface Processes and Landforms, 40(4), 2015, pp. 473-486. https://doi.org/10.1002/esp.3648

8. Barbero-García, I., Lerma, J. L., Marqués-Mateu, Á., \& Miranda, P. Low-cost smartphone-based photogrammetry for the analysis of cranial deformation in infants in World neurosurgery, vol. 102, pp. 545-554, June 2017.

9. Costanzo, A., Falcone, S., Bisignano, R., Straface, M., Ritacco, G., Piana, C. L., ... and Calimeri, F. A Smartphone Application for Supporting the Data Collection and Analysis of the Cultural Heritage Damaged during Natural Disasters, in Multidisciplinary Digital Publishing Institute Proceedings, Vol. 2, No. 3, 121, 2017.

10. Mnati, M. N., Chong, A. K., and Majeed, A. Smartphone Sensor vs Photogrammetry for Gait Analysis, in 2019 3rd International Conference on Imaging, Signal Processing and Communication (ICISPC), IEEE, 2019, pp. 130-133.

11. Kirchhöfer, M., Chandler, J., and Wackrow, R. Cultural Heritage Recording Utilising Low-Cost Closerange Photogrammetry. Geoinformatics FCE CTU, vol. 6, pp. 185-192, 2011. 
12. Shults, R. New opportunities of low-cost photogrammetry for culture heritage preservation. International Archives of the Photogrammetry, Remote Sensing and Spatial Information Sciences, 42(5/W1), 2017.

13. Boboc, R. G., Gîrbacia, F., Postelnicu, C. C., and Gîrbacia, T. Evaluation of Using Mobile Devices for 3D Reconstruction of Cultural Heritage Artifacts. In International Conference on VR Technologies in Cultural Heritage (pp. 46-59). Springer, Cham, 2018.

14. Yilmazturk, F., and Gurbak, A. E. Geometric evaluation of mobile-phone camera images for 3D information, International Journal of Optics, 2019. https://doi.org/10.1155/2019/8561380

15. Russo, M., Giugliano, A. M., \& Asciutti, M. Mobile phone imaging for $\mathbf{C H}$ façade modelling, The International Archives of Photogrammetry, Remote Sensing and Spatial Information Sciences, Vol. 42, 2019, pp. 287-294.

16. The Smartphone vs The Camera Industry. PhotographyLife.https://photographylife.com/smartpho ne-vs-camera-industry (accessed on May 2020).

17. Opto engineering, 2020. https://www.opto-e.com/basics/sensor-types-ccd-and-cm os (accessed on May 2020).

18. Bunga A. Hutagaol, Lufty Abdilah, Scelen Utari Ramona Jaya, Gunawan Wang. Adoption of Mobile Smartphone Attendance System Based on Case Study of PT XYZ, International Journal of Advanced Trends in Computer Science and Engineering, 9(1), 2020, pp.21-25. https://doi.org/10.30534/ijatcse/2020/04912020

19. Magnan, P. Detection of visible photons in CCD and CMOS: A comparative view, Nuclear Instruments and Methods in Physics Research Section A: Accelerators, Spectrometers, Detectors and Associated Equipment, 504(1-3), 2003, pp. 199-212.

20. Alfio, V. S., Costantino, D., and Pepe, M., Influence of Image TIFF Format and JPEG Compression Level in the Accuracy of the 3D Model and Quality of the Orthophoto in UAV Photogrammetry. Journal of Imaging, 6(5), 30, 2020.

21. Pepe, M., Costantino, D. and Restuccia Garofalo, A. An Efficient Pipeline to Obtain 3D Model for HBIM and Structural Analysis Purposes from 3D Point Clouds. Applied Sciences, 10(4), 1235, 2020. https://doi.org/10.3390/app10041235

22. Lindenbergh, R., Pfeifer, N. and Rabbani, T., Accuracy analysis of the Leica HDS3000 and feasibility of tunnel deformation monitoring. in Proceedings of the ISPRS Workshop, Laser scanning, Vol. 36, p. 3, September 2005. 